\title{
Estimates of Prevalence Rates of Cancer Patients With Children and Well-Being in Affected Children: A Systematic Review on Population-Based Findings
}

\author{
Laura Inhestern ${ }^{1 *}$, Johanna Christine Bultmann ${ }^{1}$, Lene Marie Johannsen ${ }^{1}$, \\ Volker Beierlein ${ }^{1}$, Birgit Möller ${ }^{2}$, Georg Romer ${ }^{3}$, Uwe Koch ${ }^{1}$ and Corinna Bergelt ${ }^{1,4}$ \\ ${ }^{1}$ Department of Medical Psychology, University Medical Center Hamburg-Eppendorf, Hamburg, Germany, ${ }^{2}$ Department of \\ Social Work, Münster University of Applied Sciences, Münster, Germany, ${ }^{3}$ Department of Child and Adolescent Psychiatry, \\ Psychotherapy and Psychosomatics, University Medical Center Münster, Münster, Germany, ${ }^{4}$ Department of Medical \\ Psychology, University Medicine Greifswald, Greifswald, Germany
}

OPEN ACCESS

Edited by:

Andreas Stengel,

Charité - Universitätsmedizin

Berlin, Germany

Reviewed by:

Norbert Schäffeler,

Tübingen University

Hospital, Germany

Pandora Patterson,

The University of Sydney, Australia

*Correspondence:

Laura Inhestern

l.inhestern@uke.de

Specialty section:

This article was submitted to

Psychosomatic Medicine,

a section of the journal

Frontiers in Psychiatry

Received: 26 August 2021

Accepted: 20 October 2021

Published: 25 November 2021

Citation:

Inhestern L, Bultmann JC, Johannsen LM, Beierlein V, Möller B,

Romer $\mathrm{G}$, Koch $\cup$ and Bergelt $C$ (2021) Estimates of Prevalence Rates of Cancer Patients With Children and Well-Being in Affected Children: A

Systematic Review on

Population-Based Findings.

Front. Psychiatry 12:765314. doi: 10.3389/fpsyt.2021.765314
This review assessed population-based estimate rates of cancer patients with minor and young adult children ( $\leq 25$ years), children and young adults having a parent with cancer as well as the psychosocial situation and well-being of children and young adults affected by parental cancer. Eighteen publications on population-based studies were included. Studies varied in the age ranges of both cancer patients and children. The prevalence rates of cancer patients having children ranged from 14 to $24.7 \%$ depending on the sample structure (e.g., age, gender). Studies reported that between 1.6 and $8.4 \%$ of children resp. young adult children have a parent with a history of cancer. Seven publications reported on the psychosocial situation or well-being in children and young adults affected by parental cancer. Estimate rates of psychosocial problems, psychiatric diagnoses or distress ranged between 2.5 and $34 \%$ of children depending on the method of measurement and outcome. The differences in the sample structure between the studies impeded the comparison of prevalence rates. However, the findings help to determine the need for specific support services and health care planning. The results emphazise the importance to routinely include issues on the parental role of patients and questions on the well-being and coping of children into psychooncological care. If necessary, support should be provided to families living with a cancer diagnosis.

Keywords: parental cancer, children, cancer, oncology, distress, well-being, prevalence

\section{INTRODUCTION}

Cancer is a pervasive stressor not only for the patients themselves but also for the whole social circle (1-3). All family members are confronted with the life-threatening diagnosis and may struggle with disruption of their daily lives. Patients with cancer parenting dependent children are emotionally burdened and experience specific stressors due to the double load of being a patient and a parent $(4,5)$. The needs as a patient can counteract with the parental role leading to feelings of insufficiency and guilt $(6,7)$. 
Children depending on their parents suffer when a parent is diagnosed with cancer and can show elevated levels of distress (8-10). Additionally, they experience cancer specific stressors such as dealing with uncertainty or concerns about the parent (11-13). Moreover, parents' capacities caring about their children emotionally and practically may be reduced due to physical and mental strains (11).

There is a growing awareness that families with dependent children having a parent with cancer are in need for tailored, specialized and systematic professional support while dealing with the destabilization and distress caused by cancer (14-16).

Recommendations and interventions providing support for affected parents and children have been published $(14,15,17)$. These mostly focus on providing help in the current situation of the parental disease and preventing long-term difficulties in the children.

Given the growing evidence that parental cancer is a risk factor for psychosocial problems in children and the fact that health care guidelines call for taking families and children of cancer patients systematically into account and provide them with appropriate services $(18,19)$, estimations of prevalence rates of cancer patients with dependent children, children having a parent with cancer and well-being of affected children are essential to organize the health care system and appropriate support services.

Until now, many studies investigating the impact of parental cancer on parents themselves or their children are based on selected samples e.g., families seeking for psychosocial support, families attending health care services, or families participating in research studies (20-23). Results based on such selective samples are systematically biased. Hence, they may not provide reliable population-based prevalence rates of cancer patients with dependent children or children affected by parental cancer. A large percentage of parents with cancer and their children, who require support, may not have access to specific services and may not use corresponding services or participate in research studies. Representative population samples must be examined in order to obtain unbiased prevalence data of numbers of affected parents and children as well as prevalence data on psychosocial situation and wellbeing in children. These data may help to determine the need for specific support services and for rigorous health care planning.

Hence, the purpose of this systematic review was to provide an overview of estimate rates on the prevalence of parents with cancer, on children having a parent with cancer and on psychosocial situation in children from populationbased studies.

\section{METHODS}

We conducted a systematic review using the PRSIMA (Preferred Reporting Items for Systematic Reviews and Meta-Analyses) guidelines (24). For preparation of the review, a protocol was developed to guide the realization of the systematic review regarding the objective and the planned methods. The protocol was not published.

\section{Search Strategy}

We searched the databases CINAHL, Medline, PsycINFO, and PSYNDEX in January 2018 with updates in April 2019, February 2020 and September 2021. We did not limit the search with regard to year of publication. Key search terms included the following terms: (prevalence* OR population-based OR epidemiology OR survey* OR incidence*) AND (parental cancer* OR parental illness* OR parental disease* OR parental physical illness* OR parent with cancer OR disabled parent*). We also conducted a systematic search of citations and references of included publications to identify further relevant studies.

\section{Eligibility Criteria}

Publications were included if they met the following criteria: accessibility of full text, published in a peer-reviewed journal, written in English or German, including data on children and young adults ( $\leq 25$ years) having a parent with cancer or including data on parental status of patients with a history of cancer, reporting population estimates of parental status of patients with cancer or population estimates of children and young adults ( $\leq 25$ years) having a parent with cancer. Publications were defined as population-based when they were carried out in a sample of the general population e.g., through sampling strategies or when they were based on data from registries (e.g., cancer registry). Publications were excluded if data was only reported for parental illness in general and not for parental cancer separately. Studies on the psychosocial situation and well-being of children and young adults affected by parental cancer were included if they reported any information on psychosocial outcomes (e.g., psychosocial problems, distress/stress levels, quality of life, internalizing or externalizing problems, psychiatric diagnoses).

\section{Study Selection}

Titles and abstracts of the publications identified were screened according to the predefined inclusion and exclusion criteria (Supplementary Material 1). When title and abstracts were relevant or when eligibility was unclear, the full text was retrieved. Identified full texts were screened based on the inclusion and exclusion criteria. If there was uncertainty about the eligibility after assessing the full text, the eligibility was discussed in the study team. The flow diagram of the selection process is presented in Figure 1.

\section{Data Extraction and Quality Assessment}

Two members of the research team performed data extraction independently using a data extraction form. The standardized form included the following information: citation details, source of information, study population, response rate, estimate/prevalence of parental cancer/affected children and if applicable, ascertainment and information on the psychosocial situation or well-being of children. In some included publications, the estimate of the prevalence or the well-being of children was not the main focus of the study but available from published information in the article.

To assess the quality of the studies two members of the research team independently rated each study using 


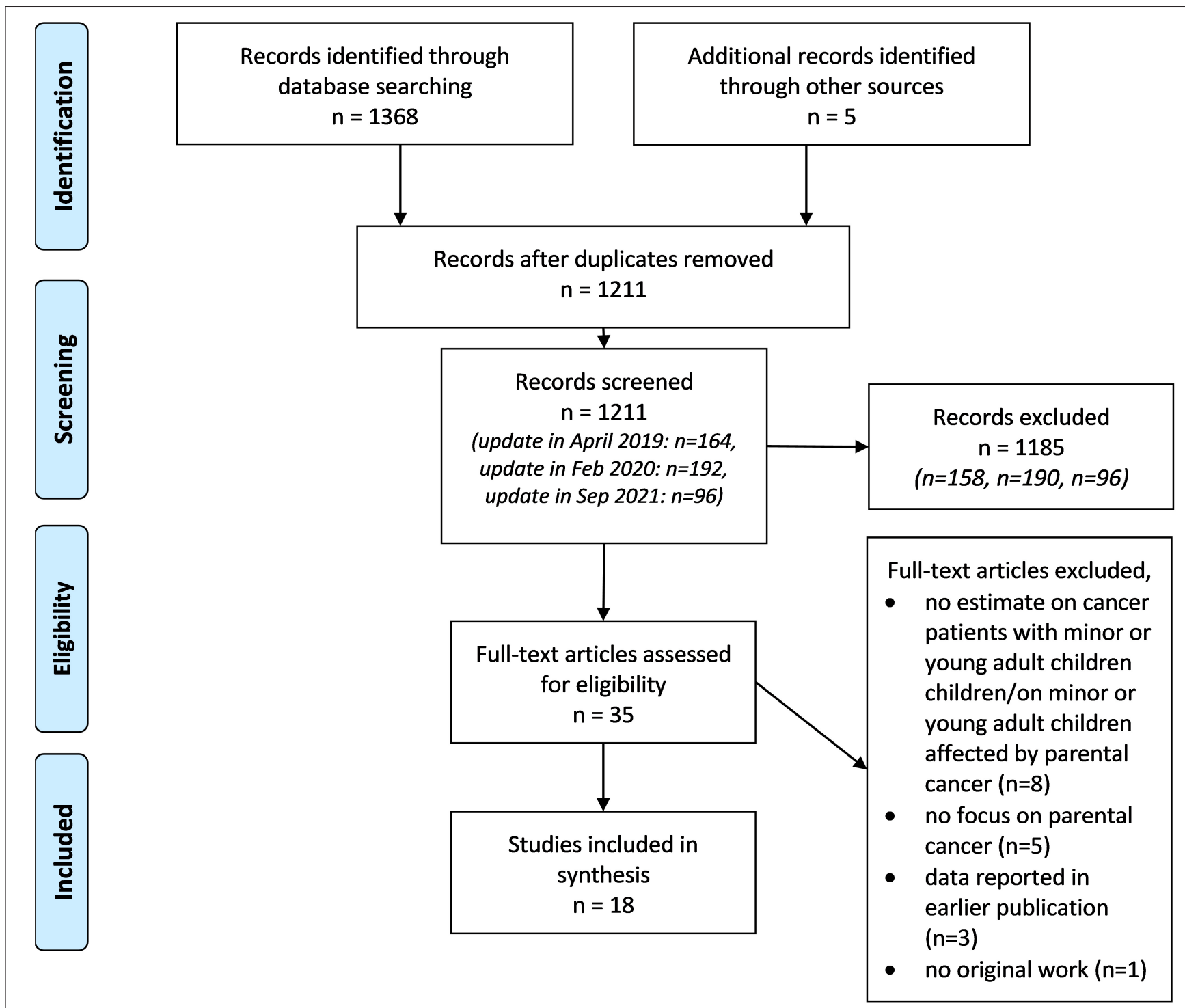

FIGURE 1 | Flow diagram for study selection and reasons for exclusion.

modified criteria adapted from the Newcastle Ottawa Scale (25). Applied criteria comprised rating on the sample selection (incl. representativeness, response rate, sample size, assessment of exposure of cancer) and, if applicable, on the assessment of well-being or the psychosocial situation of children having a parent with cancer (including assessment instrument, data source, statistical tests). All criteria could be rated as "yes," "no," "unclear," or "not applicable" (Supplementary Material 2).

\section{Analysis Strategy}

The data extracted from the included articles were synthesized descriptively and narratively. Since the included age ranges of children and/or young adults and the basic populations differ between studies, we only conducted descriptive synthesis. In case of multiple articles based on the same sample, we described the estimate rates for the article with the most complete study sample or the additional estimate rates separately (e.g., prevalence of parental cancer and information of psychosocial situation/wellbeing). Due to the heterogeneity of the characteristics of included study samples, we refrained from calculating pooled estimate rates.

\section{RESULTS}

Titles and abstracts of 1.211 articles identified from our systematic literature search were examined (Figure 1). Of these, we retrieved 35 full texts and screened them for eligibility with regard to the predefined inclusion and exclusion criteria. After the full text screening, we excluded 17 articles for several reasons (Figure 1). Three articles were excluded (26-28) since articles on the same study reported data on more complete study samples $(29,30)$. Hence, in total we included 18 articles in our synthesis. 


\section{Study Characteristics}

The 18 articles were based on 14 different samples. Three articles (three samples) reported estimate rates on the prevalence of cancer patients with minor or young adult children (30, 31), whereas 11 articles reported estimate rates on children or young adults having a parent with cancer (29, 32-41). Two articles reported both, estimate rates of cancer patients with minor or young adult children and children and young adults having a parent with cancer $(42,43)$. Three articles reported prevalence of parental cancer and prevalence on psychosocial situation and well-being in children (44-46). Since they were based on a study sample which is covered by other included articles, these three articles were only included with regard to the prevalence of psychosocial situation in children.

Most articles were from western countries (Table 1). Since most of the studies were solely based on registry data, the quality of the study was acceptable to high for the majority of included publications (Supplementary Material 2).

\section{Prevalence of Cancer Patients With Minor and Young Adult Children}

Overall, five articles reported estimates of the prevalence of cancer patients with minor or young adult children or parents with a history of cancer (Table 1). Among the publications, the age ranges of children and young adults varied (Table 1). The total sample size of cancer patients or cancer survivors included ranged from $N=8.144$ to $N=$ 151.756. Most prevalent diagnoses in cancer patients with minor or young adult children across all studies were breast and uterine/cervical cancer in mothers and cancer of the digestive organs and cancer of the prostate/testis in fathers. In the included articles mothers were more often affected by cancer than fathers.

The estimates of the prevalence of affected parents with children varied between studies. One article refers to a study based on two cancer registries from Germany with cancer survivors (25-55 years at time of the diagnosis, up to 6 years after diagnosis). At time of the diagnosis $22.4 \%$ (53.7\% of respondents) were parent of at least one child $\leq 21$ years (30).

A register-based study from Japan reported a prevalence of $24.7 \%$ of cancer patients between 20 and 59 years with minor children ( $<18$ years) (42). In a sample from Norway, $13.5 \%$ of 84.202 women with a history of cancer had minor children and $15.9 \%$ had young adult children (19-25 years); in 67.554 men with cancer $14 \%$ were fathers of minor children and $15.6 \%$ of young adult children (19-25 years) (43). Of the minor children $33 \%$ (mothers) $/ 23 \%$ (fathers) and of the young adult children, $7 \%$ had not yet been born at time of diagnosis of the parent (43).

A study from the USA reported a prevalence of $14.0 \%$ of cancer survivors with minor children, whereas in $33.5 \%$ of the cases, the children were born after diagnosis. In recently $(<2$ years prior the survey) diagnosed cancer patients, $18.3 \%$ had minor children (31).

\section{Prevalence of Minor and Young Adult Children Having a Parent With Cancer}

Thirteen articles reported estimates of children or young adults having a parent with a history of cancer. The specific age ranges of children varied between studies (Table 2). The size of the total sample ranged from $N=1.950$ to $N=3.868 .496$ children (Table 2).

In a representative survey from Germany, $4.1 \%$ of the children (4-18 years) had a physically ill parent, about $1 / 3$ of these had a parent with cancer (32). In a study including individuals with childhood psychiatric diagnosis (age at diagnosis $<15$ years), $2.2 \%$ of the children were exposed to a parental cancer diagnosis at the age between 0 and 15 years, whereas $6.8 \%$ had been exposed to parental cancer in their lifetime (33). Three articles based on different sub-populations of register-based data from Sweden reported that between 4.4 and $5.5 \%$ of the children (1-18 years) had been exposed to parental cancer $(29,34,41)$. An article based on the Finnish Birth Cohort Study 1987 identified 4.5\% of children (0-18 years) having a parent with cancer. Expanding the age range to $0-21$ years, the prevalence was $6.6 \%$ (40). A publication from Norway reported that $3.1 \%$ of the children $(0-$ 18 years) had a parent with cancer (43). In a study with teenagers, $1.6 \%$ of the participating teenagers (13-19 years) had a parent with cancer (35). In young adults (19-25 years) a prevalence of $8.4 \%$ was reported (43). A publication based on a Danish sample found that $4.3 \%$ of children between 0 and 15 years and $5.1 \%$ of children between 0 and 18 years had been exposed to parental cancer (36). Another study from Denmark focusing on maternal cancer revealed that $2.7 \%$ of children between 0 and 18 years experienced a maternal cancer diagnosis (39).

Four articles presented estimate rates on children experiencing incident cancer diagnosis of a parent annually. Estimate rates ranged between $0.28 \%(0-11$ year old children, Western Australia) (37), 0.30\% (0-18 year old children, Norway) (43), $0.38 \%$ (0-18 year old children, Japan) (43), and $0.48 \%$ (1224 year old children, Western Australia) (39) of children resp. young adults from the age-based total population experiencing a new cancer diagnosis in a parent within a 12 months period.

\section{Well-Being of Children Having a Parent With Cancer}

Seven included population-based studies reported information on the psychosocial situation or well-being of children having a parent with cancer. These aspects were assessed using different instruments and assessment strategies, which results in different prevalence rates (Table 3). Documentation of psychiatric care use and documented psychiatric diagnosis yielded prevalence rates between 2.5 and $16.2 \%(38,40,42,46)$ in different samples (e.g., regarding age, gender). One study including parental report of cancer survivors reported a prevalence rate of $10.8 \%$ of distress in children (6-18 years) up to 6 years after diagnosis parental cancer diagnosis (44). A publication on stress resilience in young adult men ( $\sim 18$ years) yielded low stress resilience in $21.4 \%$ of men with a parent with cancer (34). In a study including adolescents based on self-rated questionnaires on several psychosocial problems (e.g., self-esteem, eating problems), rates of several 
TABLE 1 | Study characteristics and estimates of parents affected with cancer from $n=5$ articles.

\begin{tabular}{|c|c|c|c|c|c|c|c|}
\hline References & Country & $\begin{array}{l}\text { Database and data } \\
\text { source }\end{array}$ & Total sample $(N)$ & $\begin{array}{l}\text { Response } \\
\text { rate }\end{array}$ & $\begin{array}{l}\text { Estimate on parents } \\
\text { with cancer and } \\
\text { children }\end{array}$ & $\begin{array}{l}\text { Characteristics of } \\
\text { parents with cancer: } \\
\text { mean age; } \\
\text { gender; } \\
\text { main diagnoses }\end{array}$ & $\begin{array}{l}\text { Characteristics of } \\
\text { children: number; } \\
\text { age; } \\
\text { gender }\end{array}$ \\
\hline Ernst et al. (30) & Germany & $\begin{array}{l}\text { Cancer registries, } \\
\text { Parental report }\end{array}$ & $\begin{array}{l}N=8.144 \text { cancer survivors } \\
\text { (exclusion of highly lethal } \\
\text { cancers: digestive and } \\
\text { lower respiratory organs, } \\
\text { brain and other parts of } \\
\text { CNS), age at diagnosis: } \\
25-55 \text { years }\end{array}$ & $41.4 \%$ & $\begin{array}{l}53.7 \% \text { ( } n=1.809 \text { ) of } \\
3.370 \text { respondents had } \\
\text { at least one child } \leq 21 \\
\text { years at time of the } \\
\text { diagnosis ( } 22.4 \% \text { of } \\
8.144 \text { potentially } \\
\text { eligible participants) }\end{array}$ & $\begin{array}{l}\text { Mean age: } 49 \text { years; } \\
\text { gender: } 74 \% \text { female; } \\
\text { main diagnoses: > } \\
50 \% \text { breast cancer }\end{array}$ & $\mathrm{NR}^{\ddagger}$ \\
\hline Inoue et al. (42) & Japan & $\begin{array}{l}\text { Cancer registry and } \\
\text { medical records }\end{array}$ & $\begin{array}{l}23.185 \text { cancer patients } \\
\text { admitted to Hospital } \\
\text { (between Jan } 2009 \text { and Dec } \\
\text { 2013), } 8.412 \text { between } 20 \\
\text { and } 59 \text { years, exclusion of } \\
1.722 \text { (no linkage possible, } \\
\text { in situ carcinoma), age: } \\
\text { 20-59 years }\end{array}$ & $N A^{\dagger}$ & $\begin{array}{l}24.7 \%(n=1.650) \text { of } \\
6.693 \text { cancer patients } \\
\text { had minor children }\end{array}$ & $\begin{array}{l}\text { Mean age: } 46.6 \text { years } \\
\text { (fathers), } 43.7 \text { years } \\
\text { (mothers); gender: } \\
55.7 \% \text { female } \\
\text { Main diagnoses: } \\
\text { gastric, lung, colorectal } \\
\text { cancer (fathers), breast, } \\
\text { uterus, gastric } \\
\text { cancer (mothers) }\end{array}$ & $\begin{array}{l}n=2.593 \text { children age: } \\
0-17 \text { years, } M=11.2 \\
\text { years gender: } \mathrm{NR}^{\ddagger}\end{array}$ \\
\hline Syse et al. (43) & Norway & $\begin{array}{l}\text { National population } \\
\text { registry and cancer } \\
\text { registry }\end{array}$ & $\begin{array}{l}84.202 \text { women with cancer, } \\
67.554 \text { men with cancer, } \\
\text { age: } 17-70 \text { years }\end{array}$ & $\mathrm{NA}^{\dagger}$ & $\begin{array}{l}13.5 \%(n=11.374) \\
\text { women with cancer } \\
\text { had minor children, } \\
14.0 \% \text { ( } n=9.469) \text { men } \\
\text { with cancer had minor } \\
\text { children; } 15.9 \% \text { ( } n= \\
\text { 13.450) women with } \\
\text { young adult children, } \\
\text { 15.6\% ( } n=10.568) \\
\text { men with young adult } \\
\text { children }\end{array}$ & $\begin{array}{l}\text { Mean age: } \mathrm{NR}^{\ddagger} ; \\
\text { gender: } 55.3 \% \text { female; } \\
\text { main diagnoses: } \\
\text { testicular, prostate, } \\
\text { colorectal cancer } \\
\text { (fathers), breast, } \\
\text { uterine/cervical cancer } \\
\text { (mothers) }\end{array}$ & $\begin{array}{l}n=34.677(<18 \\
\text { years); } n=31.592 \\
(19-25 \text { years) age: } \\
\text { minors 0-18 years } \\
(33 / 23 \% \text { not yet born } \\
\text { at diagnosis), young } \\
\text { adults } 19-25 \text { years }(7 \% \\
\text { not yet born at } \\
\text { diagnosis) gender: } \mathrm{NR}^{\ddagger}\end{array}$ \\
\hline Weaver et al. (31) & USA & $\begin{array}{l}\text { Representative sample } \\
\text { of the civilian } \\
\text { non-institutionalized } \\
\text { population, Parental } \\
\text { report }\end{array}$ & $\begin{array}{l}N=17.173 \text { cancer } \\
\text { survivors, } n=13.385 \\
\text { excluding exclusively } \\
\text { non-melanoma or unknown } \\
\text { skin cancers }(n=3.788)\end{array}$ & $67.8-74.3 \%$ & $\begin{array}{l}14 \%(n=1.769) \\
\text { cancer survivors had } \\
\text { minor children; } 18.3 \% \\
(n=341) \text { recently } \\
\text { diagnosed }(<2 \text { years } \\
\text { earlier) cancer survivors } \\
\text { had minor children }\end{array}$ & $\begin{array}{l}\text { Mean age: } \mathrm{NR}^{\ddagger} ; \\
\text { gender: } 79 \% \text { female; } \\
\text { main diagnoses: } \\
\text { breast, or } \\
\text { uterine/cervical cancer, } \\
\text { and melanoma }\end{array}$ & $\begin{array}{l}n=3.193 \text {; age at } \\
\text { diagnosis: 33.5\% not } \\
\text { yet born; 30.5\% 0-5 } \\
\text { years, } 27.2 \% 6-12 \\
\text { years, } 8.9 \% 13-17 \\
\text { years gender: } 49.5 \% \\
\text { female }\end{array}$ \\
\hline
\end{tabular}

\footnotetext{
${ }^{\dagger}$ not applicable (register-based data); ${ }^{\dagger}$ not reported.
} 
TABLE 2 | Study characteristics and estimates of children and/or young adults having a parent with cancer from $n=13$ articles.

\begin{tabular}{|c|c|c|c|c|c|c|c|}
\hline References & Country & $\begin{array}{l}\text { Database and data } \\
\text { source }\end{array}$ & Total Sample $(N)$ & $\begin{array}{l}\text { Response } \\
\text { rate }\end{array}$ & $\begin{array}{l}\text { Estimate on children having a } \\
\text { parent with cancer }\end{array}$ & $\begin{array}{l}\text { Characteristics of parents } \\
\text { with cancer: age } \\
\text { Gender } \\
\text { main diagnoses }\end{array}$ & $\begin{array}{l}\text { Characteristics of } \\
\text { children: age; } \\
\text { gender }\end{array}$ \\
\hline $\begin{array}{l}\text { Barkmann et al. } \\
\text { (32) }\end{array}$ & Germany & $\begin{array}{l}\text { Representative survey, } \\
\text { Parent report }\end{array}$ & $N=1.950$ children & $73.1 \%$ & $\begin{array}{l}4.1 \%(n=79) \text { of the children had } \\
\text { a parent with a physical illness, } \\
\text { of these } 28.8 \% \text { had a parent with } \\
\text { cancer }(n=23,1.2 \% \text { of the total } \\
\text { sample) }\end{array}$ & $N R^{\ddagger}$ & $\begin{array}{l}\text { Age: } 4-18 \text { years } \\
\text { Gender: NR }\end{array}$ \\
\hline Benros et al. (33) & Denmark & $\begin{array}{l}\text { Civil registration } \\
\text { system, psychiatric } \\
\text { central register, and } \\
\text { cancer registry }\end{array}$ & $\begin{array}{l}N=61.950 \text { children with } \\
\text { psychiatric diagnosis in a } \\
\text { population-based cohort of } \\
n=3.162 .109\end{array}$ & $N A^{\dagger}$ & $\begin{array}{l}6.8 \%(n=4.342) \text { had been } \\
\text { exposed to parental cancer, } \\
2.2 \%(n=1.348) \text { were aged } \\
<15 \text { years at the time of the } \\
\text { diagnosis of the parent }\end{array}$ & $N R^{\ddagger}$ & $\begin{array}{l}\text { Age: }<15 \text { years } \\
\text { Gender: NR }\end{array}$ \\
\hline Chen et al. (29) & Sweden & $\begin{array}{l}\text { Nationwide } \\
\text { register-based cohort } \\
\text { study }\end{array}$ & $N=2.871 .242$ children & $N A^{+}$ & $\begin{array}{l}5.5 \%(n=113.555) \text { children had } \\
\text { been exposed to parental cancer }\end{array}$ & $\begin{array}{l}\text { Age: in } 75.4 \% \text { maternal age at } \\
\text { child's birth was } 20-34 \text { years } \\
\text { Gender: NR }{ }^{\ddagger} \\
\text { Main diagnoses: NR }\end{array}$ & $\begin{array}{l}\text { Age: } 1-18 \text { years } \\
\text { Gender: } 48.7 \% \text { female }\end{array}$ \\
\hline Chen et al. (34) & Sweden & $\begin{array}{l}\text { Register-based data } \\
\text { (e.g., multi-generation } \\
\text { register, conscription } \\
\text { register and cancer } \\
\text { registry) }\end{array}$ & $\begin{array}{l}N=465.249 \text { young men } \\
\text { undergoing military } \\
\text { conscription examination }\end{array}$ & $N A^{\dagger}$ & $\begin{array}{l}4.4 \%(n=20.383) \text { young men } \\
\text { had been exposed to parental } \\
\text { cancer }\end{array}$ & $\begin{array}{l}\text { Age: in } 58.9 \% \text { paternal age at } \\
\text { child's birth was } 20-34 \text { years, in } \\
77.8 \% \text { maternal age at child's } \\
\text { birth was } 20-34 \text { years } \\
\text { Gender: } \mathrm{NR}^{\ddagger} \\
\text { Main diagnoses: } \mathrm{NR}^{\ddagger}\end{array}$ & $\begin{array}{l}\text { Age: Approximately. } 18 \\
\text { years (0-17 at time of } \\
\text { parental diagnoses) } \\
\text { Gender: } 100 \% \text { male }\end{array}$ \\
\hline Inoue et al. (42) & Japan & $\begin{array}{l}\text { Register based data } \\
\text { and medical records }\end{array}$ & $\begin{array}{l}2.563 \text { minor children among } \\
1.650 \text { cancer patients; total } \\
\text { population of minors in } \\
\text { Japan in } 201022.780 .000\end{array}$ & $N A^{\dagger}$ & $\begin{array}{l}0.38 \% \text { annual incidence of } \\
\text { children being exposed to } \\
\text { parental cancer in } 2010\end{array}$ & See Table 1 & Age: $M=11.2$ years \\
\hline $\begin{array}{l}\text { Jeppesen et al. } \\
\text { (35) }\end{array}$ & Norway & $\begin{array}{l}\text { Representative sample } \\
\text { and linkage with cancer } \\
\text { registry }\end{array}$ & $\begin{array}{l}N=8.986 \text { students } \\
\text { registered in junior high and } \\
\text { high schools (13-19 years) }\end{array}$ & $88 \%$ & $\begin{array}{l}1.6 \%(n=143) \text { teenagers had } \\
\text { been exposed to parental } \\
\text { cancer; }(6 \% \text { of the parents had } \\
\text { been diagnosed with cancer } \\
\text { before the teenager had been } \\
\text { born) }\end{array}$ & $\begin{array}{l}\text { Age: fathers: } M=49 \text { years, } \\
\text { mothers: } M=45 \text { years } \\
\text { Gender: } 49.2 \% \text { female } \\
\text { Main diagnoses: melanoma } \\
(17 \%) \text {, breast }(15 \%) \text {, } \\
\text { testicular }(12 \%)\end{array}$ & $\begin{array}{l}\text { Age: } 13-19 \text { years } \\
\text { Gender: } 49.7 \% \text { female }\end{array}$ \\
\hline $\begin{array}{l}\text { Joergensen et al. } \\
\text { (36) }\end{array}$ & Denmark & Register-based data & $\begin{array}{l}\text { Study population } 1 \text { (SP 1): } N \\
=795.160 \text { children 0-15 } \\
\text { years born between } 1986 \\
\text { and 1999; Study population } \\
2 \text { (SP 2): } N=360.054 \\
\text { children 0-18 years born } \\
\text { between } 1978 \text { and } 1984\end{array}$ & $N A^{\dagger}$ & $\begin{array}{l}\text { 4.3\% }(n=34.373) \text { children had } \\
\text { been exposed to parental cancer } \\
\text { (SP 1) } \\
5.1 \% \text { ( } n=18.378) \text { children had } \\
\text { been exposed to parental cancer } \\
\text { (SP 2) }\end{array}$ & $\begin{array}{l}\text { Age: in } 68.1 \% \text { maternal age at } \\
\text { child's birth was } 25-34 \text { years, in } \\
57.2 \% \text { paternal age at child's } \\
\text { birth was } 25-34 \text { years } \\
\text { Gender: } 57.6 \% \text { female }\end{array}$ & $\begin{array}{l}\text { SP } 1 \$: \\
\text { Age: } 0-15 \text { years; } \\
\text { Gender: } 50.1 \% \text { female } \\
\text { SP } 2 \$: \\
\text { Age: } 0-18 \text { years } \\
\text { Gender: } 49.0 \% \text { female }\end{array}$ \\
\hline
\end{tabular}


TABLE 2 | Continued

\begin{tabular}{|c|c|c|c|c|c|c|c|}
\hline References & Country & $\begin{array}{l}\text { Database and data } \\
\text { source }\end{array}$ & Total Sample $(N)$ & $\begin{array}{l}\text { Response } \\
\text { rate }\end{array}$ & $\begin{array}{l}\text { Estimate on children having a } \\
\text { parent with cancer }\end{array}$ & $\begin{array}{l}\text { Characteristics of parents } \\
\text { with cancer: age } \\
\text { Gender } \\
\text { main diagnoses }\end{array}$ & $\begin{array}{l}\text { Characteristics of } \\
\text { children: age; } \\
\text { gender }\end{array}$ \\
\hline Martini et al. (37) & Australia & $\begin{array}{l}\text { Register-based data } \\
\text { and linkage with } \\
\text { genealogy databases }\end{array}$ & $\begin{array}{l}N=25.901 \text { children } \\
\text { affected by incident cancer } \\
\text { diagnosis (1982-2015) }\end{array}$ & $N A^{\dagger}$ & $\begin{array}{l}0.28 \%(n=1.149) \text { annual } \\
\text { incidence of children being } \\
\text { exposed to parental cancer in } \\
2015\end{array}$ & $\begin{array}{l}\text { Age at diagnosis: } \mathrm{M}=39.9 \\
\text { years } \\
\text { Gender: } 53 \% \text { female } \\
\text { Main diagnoses: breast, } \\
\text { melanomas, digestive organs }\end{array}$ & $\begin{array}{l}\text { Age: } 0-11 \text { years } \\
\text { Gender: } 48 \% \text { female }\end{array}$ \\
\hline Momen et al. (38) & Denmark & Register-based data & $N=2.158 .430$ children & $N A^{\dagger}$ & $\begin{array}{l}0.13 \%(n=2.725) \text { of children } \\
\text { being exposed to maternal } \\
\text { cancer prenatal, 3.69\% }(n= \\
63.862) \text { being exposed to } \\
\text { maternal cancer postnatal }\end{array}$ & $\begin{array}{l}\text { Age: maternal age in } 46 \% \\
\geq 31 \text { years (prenatal exposure); } \\
\text { maternal age in } 48 \% \text { 31years } \\
\text { (postnatal exposure) } \\
\text { Gender: } 100 \% \text { female } \\
\text { Main diagnoses: NR }\end{array}$ & $\begin{array}{l}\text { Age: } 0-18 \text { years } \\
\text { Gender: } 50 \% \text { female } \\
\text { (prenatal), } 49 \% \\
\text { female (postnatal) }\end{array}$ \\
\hline Morris et al. (39) & Australia & $\begin{array}{l}\text { Register-based data } \\
\text { and linkage with } \\
\text { census data }\end{array}$ & $\begin{array}{l}N=57.708 \text { adolescents } \\
\text { and young adults (12-24 } \\
\text { years) affected by incident } \\
\text { cancer diagnosis } \\
(1982-2015)\end{array}$ & $N A^{\dagger}$ & $\begin{array}{l}0.47 \% \text { annual incidence of } \\
\text { adolescents and young adults } \\
\text { being exposed to parental } \\
\text { cancer }\end{array}$ & $\begin{array}{l}\text { Age at diagnosis: } M=51.3 \\
\text { Gender: } 47.2 \% \text { Female }\end{array}$ & $\begin{array}{l}\text { Age at parent } \\
\text { diagnosis: } \mathrm{M}=18.8 \\
\text { Gender: } 48.8 \% \text { female }\end{array}$ \\
\hline Niemelä et al. (40) & Finland & $\begin{array}{l}\text { Finnish Birth Cohort } \\
\text { Study and linkage with } \\
\text { register-based data }\end{array}$ & $N=59.476$ children & $N A^{\dagger}$ & $\begin{array}{l}6.6 \%(n=3.909) \text { had been } \\
\text { exposed to parental cancer at } \\
\text { time of diagnosis } 4.5 \% \text { of the } \\
\text { children were under the age of } \\
18 \text { years }\end{array}$ & $\begin{array}{l}\text { Age: maternal age at child's birth } \\
\mathrm{M}=31.4 \text { years } \\
\text { Gender: } 60.7 \% \text { female } \\
\text { Main diagnoses: } \mathrm{NR}^{\ddagger}\end{array}$ & $\begin{array}{l}\text { Age: } 21 \text { years at follow } \\
\text { up } \\
\text { Gender: } 48.5 \% \text { female }\end{array}$ \\
\hline Syse et al. (43) & Norway & Register-based data & $\begin{array}{l}n=34.677 \text { children }(<18 \\
\text { years) among } 20.843 \\
\text { cancer patients; } n=31.592 \\
\text { young adults }(19-25 \text { years }) \\
\text { among } 24.018 \text { cancer } \\
\text { patients }\end{array}$ & $\mathrm{NA}^{+}$ & $\begin{array}{l}3.1 \%(n=34.250) \text { minor children } \\
\text { had been exposed to parental } \\
\text { cancer, } 8.4 \% \text { ( } n=30.769) \text { young } \\
\text { adult children had been exposed } \\
\text { to parental cancer, } 0.3 \% \text { annual } \\
\text { incidence of families with minor } \\
\text { children being exposed to } \\
\text { parental cancer in } 2007\end{array}$ & see Table 1 & $\begin{array}{l}\text { Age: minors 0-18 } \\
\text { years, young adults } \\
\text { 19-25 years } \\
\text { Gender: } \mathrm{NR}^{\ddagger}\end{array}$ \\
\hline $\begin{array}{l}\text { Verkooijen et al. } \\
\text { (41) }\end{array}$ & Sweden & Register-based data & $\begin{array}{l}N=3.868 .496 \text { children } \\
\text { born between } 1960 \text { and } \\
2002\end{array}$ & $N A^{\dagger}$ & $\begin{array}{l}4.5 \%(n=174.893) \text { children had } \\
\text { been exposed to maternal } \\
\text { cancer; }\end{array}$ & $\begin{array}{l}\text { Age: maternal age at child's birth } \\
\text { between } 16-46 \text { years; } \\
\text { Gender: } 100 \% \text { female } \\
\text { Main diagnoses: breast, } \\
\text { reproductive, } \\
\text { digestive, melanoma }\end{array}$ & $\begin{array}{l}\text { Age at diagnosis: } 4.2 \% \\
1-18 \text { years, } 0.05 \% \\
\text { born } \pm 1 \text { year, } 0.2 \% \\
\text { not yet born } \\
\text { Gender: } \mathrm{NR}^{\ddagger}\end{array}$ \\
\hline
\end{tabular}

${ }^{\dagger}$ not applicable (register-based data); ${ }^{\ddagger}$ not reported; ${ }^{\S}$ study population. 
TABLE 3 | Study characteristics and estimates of psychosocial situation or well-being of children and/or young adults having a parent with cancer from $n=7$ articles

\begin{tabular}{|c|c|c|c|c|c|}
\hline Author, year & $\begin{array}{l}\text { Database and data } \\
\text { source }\end{array}$ & Sample & $\begin{array}{l}\text { Characteristics of children: } \\
\text { age; } \\
\text { gender }\end{array}$ & $\begin{array}{l}\text { Assessment of psychosocial } \\
\text { situation or well-being }\end{array}$ & $\begin{array}{l}\text { Information on well-being or } \\
\text { psychosocial outcome in children } \\
\text { having a parent with cancer }\end{array}$ \\
\hline Bultmann et al. (44) & $\begin{array}{l}\text { Cancer registries, } \\
\text { Parental report }\end{array}$ & $\begin{array}{l}n=1,449 \text { children of } n \\
=976 \text { cancer survivors }\end{array}$ & $\begin{array}{l}\text { Age: } M=13.0 \text { years (range } 6-18 \\
\text { years); } \\
\text { Gender: } 47.9 \% \text { female }\end{array}$ & $\begin{array}{l}\text { Single item on distress (6-point-likert } \\
\text { scale, cut off } \geq 3=\text { distressed), Health } \\
\text { related quality of life (Kidscreen-10 } \\
\text { Index) }\end{array}$ & $\begin{array}{l}10.8 \% \text { of the children were currently } \\
\text { distressed; Overall: Higher HRQoL in } \\
\text { children affected by parental cancer } \\
\text { compared to norm population }\end{array}$ \\
\hline Chen et al. (34) & Register-based data & $\begin{array}{l}N=1.791 .565 \text { children } \\
\text { (born between } 1983 \\
\text { and 2000) }\end{array}$ & $\begin{array}{l}\text { Age: } 0-27 \text { years } \\
\text { Gender: } 52.8 \% \text { male }\end{array}$ & $\begin{array}{l}\text { Diagnosis of psychiatric disorder } \\
\text { (ICD-Code) }\end{array}$ & $\begin{array}{l}8.7 \% \text { (male, cancer during } \\
\text { pregnancy: } 1 \% \text {, female, cancer } \\
\text { during pregnancy: } 1.2 \% \text {; male, } \\
\text { cancer after birth: } 7.6 \%, \text { female, } \\
\text { cancer: after birth } 9.8 \% \text { ); higher risk } \\
\text { of psychiatric disorder diagnoses in } \\
\text { children of parents with cancer }\end{array}$ \\
\hline Chen et al. (34) & Register-based data & $\begin{array}{l}N=465.249 \text { young } \\
\text { men undergoing } \\
\text { military conscription } \\
\text { examination }\end{array}$ & $\begin{array}{l}\text { Age: approximately } 18 \text { years } \\
(0-17 \text { at time of parental } \\
\text { diagnoses) } \\
\text { Gender: } 100 \% \text { male }\end{array}$ & $\begin{array}{l}\text { Semi-structured interview to assess } \\
\text { ability to cope with psychological } \\
\text { stress during military service (Stanine } \\
\text { scale; score of } 1-3=\text { low stress } \\
\text { resilience) }\end{array}$ & $\begin{array}{l}21.4 \% \text { had low stress resilience; } \\
\text { higher risk for low stress resilience in } \\
\text { children of parents with cancer }\end{array}$ \\
\hline Jeppesen et al. (35) & $\begin{array}{l}\text { Representative sample } \\
\text { and linkage with cancer } \\
\text { registry }\end{array}$ & $\begin{array}{l}N=8.986 \text { students } \\
\text { registered in junior high } \\
\text { and high schools } \\
\text { (13-19 years) }\end{array}$ & $\begin{array}{l}\text { Age: } 13-19 \text { years } \\
\text { Gender: } 49.7 \% \text { female }\end{array}$ & $\begin{array}{l}\text { Several items/instruments assessing } \\
\text { different aspects of psychosocial } \\
\text { problems (somatic stress symptoms, } \\
\text { cut off } \geq 2 \text { symptoms; feeling lonely, } \\
1=\text { very often/often; eating problems } \\
\text { (EAT } 7 \text {, cut off >4); self-esteem, 0-6 } \\
=\text { low self-esteem; anxiety and } \\
\text { depression (SCL-5, cut off } \geq 2 \text { ), } \\
\text { school problems, } 1 \text { = one or more } \\
\text { problems; psychosocial problems, } \\
\text { cut off } \geq 2 \text { ); }\end{array}$ & $\begin{array}{l}10 \% \text { eating problems, } 6 \% \text { feeling } \\
\text { lonely, } 20 \% \text { low self-esteem, } \\
18 \% \text { anxiety/depression, } 41 \% \text { school } \\
\text { problems, } 36 \% \text { somatic stress } \\
\text { symptoms, high problem cases } 34 \% \text {; } \\
\text { no differences between children of } \\
\text { parents with cancer and control group }\end{array}$ \\
\hline Momen et al. (38) & Register-based data & $\begin{array}{l}N=2,725 \text { children } \\
\text { (prenatal exposure to } \\
\text { cancer) } \\
N=63.708 \text { children } \\
\text { (postnatal exposure } \\
\text { to cancer) }\end{array}$ & $\begin{array}{l}\text { Age: } 0-18 \text { years } \\
\text { Gender: } 50 \% \text { female (prenatal } \\
\text { exposure), } 49 \% \text { female } \\
\text { (postnatal exposure) }\end{array}$ & $\begin{array}{l}\text { Psychiatric diagnoses according to } \\
\text { ICD-Code }\end{array}$ & $\begin{array}{l}3.4 \%(n=93, \text { prenatal exposure to } \\
\text { cancer), } 2.5 \%(n=1,584 \text {, postnatal } \\
\text { exposure to cancer) }\end{array}$ \\
\hline Niemelä et al. (40) & $\begin{array}{l}\text { Finnish Birth Cohort } \\
\text { Study and linkage with } \\
\text { register-based data }\end{array}$ & $N=59.476$ children & $\begin{array}{l}\text { Age: } 21 \text { years at follow up } \\
\text { Gender: } 48.5 \% \text { female }\end{array}$ & Use of specialized psychiatric care & $\begin{array}{l}16.2 \% \text { had received specialized } \\
\text { psychiatric care }\end{array}$ \\
\hline Niemelä et al. $(16,46)$ & $\begin{array}{l}\text { Finnish Birth Cohort } \\
\text { Study and linkage with } \\
\text { register-based data }\end{array}$ & $N=59.476$ children & $\begin{array}{l}\text { Age: } 21 \text { years at follow up } \\
\text { Gender: } 48.5 \% \text { female }\end{array}$ & $\begin{array}{l}\text { Use of psychiatric outpatient care \& } \\
\text { psychiatric diagnoses according to } \\
\text { ICD-Code }\end{array}$ & $\begin{array}{l}\text { In total, } 14 \% \text { had received psychiatric } \\
\text { outpatient care, } 10.8 \% \text { had been } \\
\text { diagnosed with a mental disorder }\end{array}$ \\
\hline
\end{tabular}

Representative sample and linkage with cancer registered in junior high and high schools

EAT, Eating Attitude Test; SCL, Symptom Check List.

$N=63.708$ children

o cancer)
Finnish Birth Cohort $\quad N=59.476$ children

Study and linkage with
,725 children

exposure), $49 \%$ female 
symptoms were between 6 (feeling lonely) and 41\% (school problems). Summarizing the scales, $34 \%$ of adolescents yielded as high total problem cases (35).

\section{DISCUSSION}

Cancer invades and causes ripple effects in the family. This narrative review compiles the prevalence rates of parental cancer from estimations of various population-based studies. Overall, the estimate rates varied among the populations due to different response rates or different characteristics of the included populations (e.g., age, gender). Eighteen publications met our inclusion criteria and of those, seven publications also reported information on the psychosocial situation or well-being of children having a parent with cancer.

The population-based study samples included between 8.144 and 151.756 cancer patients or cancer survivors and between 1.950 and 3.868.496 children. This review shows that cancer affects a considerable part of parents with between 14 and $24.7 \%$ of cancer patients having minor children, depending on age range of the sample.

In the future possibly even more children may experience parental cancer, since screenings programs may detect cancer earlier and more patients survive or live with cancer for many years due to treatment. Moreover, many people choose to have children later in life these days: in Germany about $27 \%$ of all women giving birth are older than 35 years (47). The mean age of having the first child in EU countries is about 29.1 years (48). Additionally, the transition to adulthood (leaving home) has shifted to later life: In the EU, men move from home at a mean age of 27 years and women at mean age of 25 years (49). Taking this into account, makes it even more likely that, in future, cancer will be diagnosed more frequently among patients caring for minor and young adult children or living with young adult children in the same household.

The prevalence rates of children, adolescents and young adult children having a parent with a history of cancer range from 1.6 to $8.4 \%$ depending on the sample and age range taken into account. Studies on the well-being of children affected by parental cancer report estimate rates between 2.5 and $34 \%$ of children with substantial psychosocial burden. Compared to populations not being affected by parental cancer, the findings show mixed results. Some of the included articles found a slightly higher risk of a psychiatric diagnosis in children of parents with cancer compared to matched controls $(34,40,45,46)$, while other articles found no overall differences $(35,38)$. One article on quality of life found higher parent-reported quality of life in children of cancer survivors compared to norm values (44).

These findings indicate that children may not generally be at risk for psychopathological meaningful repercussions of the parental disease. As a matter of fact, studies in non-populationbased samples of children affected by parental cancer identify higher distress and anxiety as well as lower quality of life compared to normative values $(50,51)$ and about one third of a sample of adolescents reported psychosocial problems (52). However, these samples were highly selective e.g., recruited among users of psychosocial support offers or in the context of cancer care for the parents.

Factors mediating the impact of parental cancer are e.g., family functioning or characteristics of the disease (11). Parents may provide adequate support to their children and families deal with a parental cancer diagnosis in an appropriate way, e.g., by communicating with their children openly or maintaining daily routines $(53,54)$. Moreover, families may have resources, which they use to cope with the situation and encounter the challenges of the parental disease $(4,55,56)$.

While few publications in this review included questionnaires on psychosocial problems or stress based on proxy reports, most studies on estimate rates of psychological consequences do refer to psychiatric diagnoses based on data from health registries using DSM or ICD criteria. However, psychopathology might not be an appropriate indicator to identify psychosocial problems or burden of children facing parental cancer. Children may rather experience cancer specific concerns and fears, which may not be captured by general psychopathology since they might cause substantial mental burden, but do not meet the diagnostic criteria of psychiatric diseases $(11,57)$. Qualitative studies on children of cancer patients and studies using selected samples and more general instruments to detect stress symptoms or quality of life, indicate that children are experiencing substantial emotional burden, and hence, can benefit from supportive counseling (11, 14). Interventions to support children with a parent with cancer have been developed and shown to be valuable and helpful for children to cope with the potentially life-threatening disease of their parent and to deal with their own emotions $(17,58)$. Still, in current health care, minor children are not routinely included in health care and cooperation with child mental health care providers, as a preventive support offer, is rare (16).

Our findings on prevalence rates on affected parents and children underline the importance to routinely (1) assess the familial background of a patient and circulate this information within the health-care team and (2) assess the psychosocial situation of minor and young adult children as relatives of the patients. Our results reveal the necessity of an assessment of the psychosocial situation not only during acute treatment phase but also in aftercare. Where needed, psychosocial care for affected parents and children should be offered to all family members $(18,19)$.

\section{Limitations}

Looking at the results of this review, some limitations need to be considered. Our primary interest was to review publications conducted in population-based studies. However, the results must be interpreted with caution since we included heterogeneous studies with different approaches and samples to estimate prevalence rates and used different criteria to assess mental health outcomes. The quality of included studies differs with regard to representativeness which e.g., might be caused by different sampling strategies and methodology. Indeed, the inclusion of population-based study reduces potential selection bias. But while some studies referred to patient and family registries with nearly complete data, other studies might have suffered from a selection bias due to incomplete response rates. 
All included studies were carried out in industrial countries and we cannot draw any conclusions with regard to developing countries. The strong difference in study populations hampers the possibility to provide an overall mean estimate for prevalence rates. Still, register-based data and some studies including nearly complete data allow for more precise estimates on prevalence rates than studies based on selected samples.

Results on the psychosocial situation and well-being of children affected by parental cancer should be interpreted with caution since not all studies included a control or comparison group from non-cancer samples. Moreover, cancer-specific burden may not be covered by the methods used in the included studies. Comparisons with non-population-based samples on children affected by parental cancer could not be conducted systematically due to incoherent use of assessment instruments across studies.

\section{CONCLUSIONS}

Taking into account the substantial prevalence rates of cancer patients with minor children and the increasing survival rates of cancer patients during the past decades, it is important (1) to assess the familial background of the patients and (2) to support families in living with cancer and its consequences, e.g., fatigue, fears during after-care checkups, or limited capacity due to long-term side effects. Our review obtains an overview on prevalence data of cancer patients parenting minor or young adult children and children having a parent with cancer and, hence, help to determine the need for specific support services and health care planning. As between 14 and $22 \%$ of the cancer populations in this review were parents of minor and young adult children, expertise in disease coping and developmental aspects for children should be routinely integrated in psychosocial and psycho-oncological care teams.

Our findings suggest that children are not generally at risk for negative mental long-term consequences and that children may adjust well to parental cancer. Still, instruments to assess disease specific burden in children are lacking. Future studies

\section{REFERENCES}

1. Visser A, Huizinga GA, van der Graaf WTA, Hoekstra HJ, HoekstraWeebers JEHM. The impact of parental cancer on children and the family: a review of the literature. Cancer Treat Rev. (2004) 30:68394. doi: 10.1016/j.ctrv.2004.06.001

2. Edwards B, Clarke V. The psychological impact of a cancer diagnosis on families: the influence of family functioning and patients' illness characteristics on depression and anxiety. Psychooncology. (2004) 13:56276. doi: 10.1002/pon.773

3. Hyde MK, Legg M, Occhipinti S, Lepore SJ, Ugalde A, Zajdlewicz $\mathrm{L}$, et al. Predictors of long-term distress in female partners of men diagnosed with prostate cancer. Psycho-Oncol. (2018) 27:946-54. doi: 10.1002/ pon. 4617

4. Semple CJ, McCance T. Experience of parents with head and neck cancer who are caring for young children. $J$ Adv Nurs. (2010) 66:1280-90. doi: 10.1111/j.1365-2648.2010.05 311.x should develop or refer to existing cancer-specific assessment tools. Moreover, the use of broadly used instruments on healthrelated quality of life or psychosocial problems may allow for comparisons with norm values as well as other disease groups.

Since every age group of children experiences different developmental aspects and tasks, a more distinct consideration of child age in future studies would be useful to provide a better understanding of needs during childhood. Longitudinal research including stage of illness, developmental stage of the children, and cancer-specific instruments to assess distress and needs are important and beneficial to investigate and understand the impact of parental cancer on parents as well as on children more thoroughly.

\section{DATA AVAILABILITY STATEMENT}

The original contributions presented in the study are included in the article/Supplementary Material, further inquiries can be directed to the corresponding author/s.

\section{AUTHOR CONTRIBUTIONS}

LI, LJ, and JB were involved in the data acquisition, data analysis, interpretation, and manuscript preparation. All authors were involved in the conception of the study, editing, contributed substantially to this manuscript, and reviewed the final version of this manuscript.

\section{FUNDING}

This work was supported by German Cancer Aid (grant \# 108303).

\section{SUPPLEMENTARY MATERIAL}

The Supplementary Material for this article can be found online at: https://www.frontiersin.org/articles/10.3389/fpsyt. 2021.765314/full\#supplementary-material
5. Rauch PK, Muriel AC. The importance of parenting concerns among patients with cancer. Crit Rev Oncl Hematol. (2004) 49:37-42. doi: 10.1016/S1040-8428(03)00095-7

6. Davey MP, Bilkins B, Diamond G, Willis AI, Mitchell EP, Davey A, et al. African American patients' psychosocial support needs and barriers to treatment: patient needs assessment. J Cancer Educ. (2016) 31:4817. doi: 10.1007/s13187-015-0861-9

7. Faulkner RA, Davey M. Childr and adolescents of cancer patients: the impact of cancer on the family. Am J Fam Ther. (2002) 30:6372. doi: 10.1080/019261802753455651

8. Gazendam-Donofrio SM, Hoekstra HJ, van der Graaf WTA, van de Wiel HBM, Visser A, Huizinga G, et al. Adolescents' emotional reactions to parental cancer: effect on emotional and behavioral problems. J Pediatr Psychol. (2011) 36:346-59. doi: 10.1093/jpepsy/jsq090

9. Visser A, Huizinga GA, Hoekstra HJ, van der Graad WTA, GazedamDonofrio SM, Hoekstra-Weebers JE. Emotional and behavioral problems in children of parents recently diagnosed with cancer: a longitudinal study. Acta Oncol. (2007) 46:67-76. doi: 10.1080/02841860600949560 
10. Thastum M, Watson M, Kienbacher C, Piha J, Steck B, Zachariae R, et al. Prevalence and predictors of emotional and behavioural functioning of children where a parent has cancer: a multinational study. Cancer. (2009) 115:4030-9. doi: 10.1002/cncr.24449

11. Morris JN, Martini A, Preen D. The well-being of children impacted by a parent with cancer: an integrative review. Support Care Cancer. (2016) 24:3235-51. doi: 10.1007/s00520-016-3259-2

12. Phillips F, Lewis FM. The adolescent's experience when a parent has advanced cancer: a qualitative inquiry. Palliat Med. (2015) 29:8518. doi: 10.1177/0269216315578989

13. Kennedy VL, Lloyd-Williams M. How children cope when a parent has advanced cancer. Psycho-Oncol. (2009) 18:886-92. doi: 10.1002/pon.1455

14. Ellis SJ, Wakefield CE, Antill G, Burns M, Patterson P. Supporting children facing a parent's cancer diagnosis: a systematic review of children's psychosocial needs and existing interventions. Eur J Cancer Care. (2017) 26:e12432. doi: 10.1111/ecc.12432

15. Phillips F, Prezio EA. Wonders \& worries: evaluation of a child centered psychosocial intervention for families who have a parent/primary caregiver with cancer. Psycho-Oncol. (2017) 26:1006-12. doi: 10.1002/pon.4120

16. Niemelä M, Marshall CA, Kroll T, Curran M, Silverberg Koerner S, Räsänen S, et al. Family-focused preventive interventions with cancer cosurvivors: a call to action. Am J Public Health. (2016) 106:13817. doi: 10.2105/AJPH.2016.303178

17. Niemelä M, Hakko $H$, Räsänen $S$. A systematic narrative review of the studies on structured child-centred interventions for families with a parent with cancer. Psychooncology. (2010) 19:451-61. doi: 10.1002/pon.1620

18. National Breast Cancer Centre and National Cancer Control Initiative. Clinical Practice Guidelines for the Psychosocial Care of Adults with Cancer. Camperdown, NSW: National Breast Cancer Centre (2003).

19. National Compehensive Cancer Network. NCCN Guidelines for Patients: $(\mathbb{2}$ Distress Management (2017). Retrieved from https://www.nccn.org/ guidelines/patients

20. Möller B, Barkmann C, Krattenmacher T, Kühne F, Bergelt C, Beierlein V, et al. Children of cancer patients: prevalence and predictors of emotional and behavioral problems. Cancer. (2014) 120:2361-70. doi: 10.1002/cncr.28 644

21. Huizinga GA, van der Graaf WTA, Visser A, Dijkstra JS, Hoekstra-Weebers JEHM. Psychosocial consequences for children of a parent with cancer: a pilot study. Cancer Nurs. (2003) 26:195-202. doi: 10.1097/00002820-200306000-00004

22. Krattenmacher T, Kühne F, Halverscheid S, Wiegand-Grefe S, Bergelt C, Romer G, et al. A comparison of the emotional and behavioral problems of children of patients with cancer or a mental disorder and their association with parental quality of life. J Psychosom Res. (2004) 73:21320. doi: 10.1016/j.jpsychores.2013.11.020

23. Visser A, Huizinga GA, Hoekstra HJ, van der Graaf WTA, Klip E, Pras E, et al. Emotional and behavioural functioning of children of a parent diagnosed with cancer: a cross-informant perspective. Psychooncology. (2005) 14:746-58. doi: 10.1002/pon.902

24. Moher D, Liberati A, Tetzlaff J, Altmann DG. Preferred reporting items for systematic reviews and meta-analyses: the PRISMA statement. Ann Intern Med. (2009) 15:264-9. doi: 10.7326/0003-4819-151-4-20090818000135

25. Wells GA, Shea B, O'Connell D, Peterseon J, Welch B, Losos M, et al. The Newcastle-Ottawa Scale (NOS) for Assessing the Quality of Non Randomised Studies in Meta-Analyses. (2001). Available online at: http://www.ohri.ca/ programs/clinical_epidemiology/oxford.asp (accessed April 25, 2019).

26. Inhestern L, Bultmann JC, Beierlein V, Möller B, Romer G, Koch $\mathrm{U}$, et al. Understanding parenting concerns in cancer survivors with minor and young-adult children. J Psychosom Res. (2016) 87:1-6. doi: 10.1016/j.jpsychores.2016.05.008

27. Inhestern L, Bultmann JC, Beierlein V, Möller B, Romer G, Muriel AC, et al. Psychometric properties of the parenting concerns questionnaire in cancer survivors with minor and young adult children. Psycho-Oncol. (2016) 25:1092-8. doi: 10.1002/pon.4049

28. Chen R, Regodon Wallin A, Sjölander A, Valdimarsdóttir U, Ye W, Tiemeier $\mathrm{H}$, et al. Childhood injury after a parental cancer diagnosis. eLife. (2015) 4:31. doi: 10.7554/eLife.08500
29. Chen R, Sjölander A, Valdimarsdóttir U, Varnum C, Almquist C, Ye W, et al. Parental cancer diagnosis and child mortality - a population-based cohort study in Sweden. Cancer Epidemiol. (2015) 39:79-85. doi: 10.1016/j.canep.2014.11.011

30. Ernst JC, Beierlein V, Romer G, Möller B, Koch U, Bergelt C. Use and need for psychosocial support in cancer patients: a population-based sample of patients with minor children. Cancer. (2013) 119:2333-41. doi: 10.1002/cncr.28021

31. Weaver KE, Rowland JH, Alfano CM, McNeel TS. Parental cancer and the family: a population-based estimate of the number of US cancer survivors residing with their minor children. Cancer. (2010) 116:4395401. doi: 10.1002/cncr.25368

32. Barkmann C, Romer G, Watson M, Schulte-Markwort M. Parental physical illness as a risk for psychosocial maladjustment in children and adolescents: epidemiological findings from a national survey in Germany. Psychosomatics. (2007) 48:476-81. doi: 10.1176/appi.psy.48.6.476

33. Benros ME, Laursen TM, Dalton SO, Nordentoft M, Mortensen PB. The risk of schizophrenia and child psychiatric disorders in offspring of mothers with lung cancer and other types of cancer: a Danish nationwide register study. PLoS ONE. (2013) 8:e79031. doi: 10.1371/journal.pone.0079031

34. Chen R, Fall K, Czene K, Kennedy B, Valdimarsdóttir U, Fang F. Impact of parental cancer on IQ, stress resilience, and physical fitness in young men. Clin Epidemiol. (2018) 10:593-604. doi: 10.2147/CLEP.S152210

35. Jeppesen E, Bjelland I, Fossa SD, Loge JH, Dahl AA. Psychosocial problems of teenagers who have a parent with cancer: a population-based case-control study (Young-HUNT Study). J Clin Oncol. (2013) 31:4099104. doi: 10.1200/JCO.2013.50.7061

36. Joergensen AC, Kjaer Urhoj S, Nybo Andersen AM. Primary school achievement and socioeconomic attainment in individuals affected by parental cancer in childhood or adolescence: a Danish nationwide register-based study. J Epidemiol Community Health. (2018) 72:9829. doi: 10.1136/jech-2018-210472

37. Martini A, Morris JN, Jackson HM, Ohan JL. The impact of parental cancer on preadolescent children (0-11 years) in Western Australia: a longitudinal population study. Support Care Cancer. (2019) 27:122936. doi: 10.1007/s00520-018-4480-y

38. Momen NC, Ernst A, Arendt LH, Olsen J, Li J, Gissler M, et al. Mental and behavioural disorders in the children of mothers diagnosed with cancer: a Danish population-based register study. Psycho-Oncol. (2019) 28:40814. doi: 10.1002/pon.4958

39. Morris JN, Zajac I, Turnbull D, Preen S, Patterson P, Martini A. A longitudinal investigation of Western Australian families impacted by parental cancer with adolescents and young adult offspring. Aust N Z J Public Health. (2019) 43:261-6. doi: 10.1111/1753-6405.12885

40. Niemelä M, Paananen R, Hakko H, Merikukka M, Gissler M, Räsänen S. The prevalence of children affected by parental cancer and their use of specialized psychiatric services: the 1987 Finnish Birth Cohort study. Int J Cancer. (2012) 131:2117-25. doi: 10.1002/ijc.27466

41. Verkooijen HM, Ang JX, Liu J, Czene K, Salim A, Hartmann M. Mortality among offspring of women diagnosed with cancer: a population-based cohort study. Int J Cancer. (2013) 132:2432-8. doi: 10.1002/ijc.27899

42. Inoue I, Higashi T, Iwamoto M, Heiney SP, Tamaki T, Osawa K, et al. A national profile of the impact of parental cancer on their children in Japan. Cancer Epidemiol. (2015) 39 838-41. doi: 10.1016/j.canep.2015.10.005

43. Syse A, Aas GB, Loge JH. Children and young adults with parents with cancer: a population-based study. Clinical Epidemiol. (2012) 4:4152. doi: 10.2147/CLEP.S28984

44. Bultmann JC, Beierlein V, Romer G, Möller B, Koch U, Bergelt C. Parental cancer: health-related quality of life and current psychosocial support needs of cancer survivors and their children. Int J Cancer. (2014) 135:266877. doi: 10.1002/ijc. 28905

45. Chen R, Regodon Wallin A, Selinus EN, Sjölander A, Fall K, Valdimarsdóttir $\mathrm{U}$, et al. Psychiatric disorders among children of parents with cancer: a Swedish register-based matched cohort study. Psycho-Oncol. (2018) 27:185460. doi: 10.1002/pon.4738

46. Niemelä M, Paananen R, Hakko H, Merikukka M, Gissler M, Räsänen S. Mental disorder diagnoses of offspring affected by parental cancer before early adulthood: the 1987 Finnish Birth Cohort study. Psycho-Oncol. (2016) 25:1477-84. doi: 10.1002/pon.4088 
47. Statistisches Bundesamt. Anzahl der Geburten nach dem Alter der Mutter in Deutschland im Jahr 2017. (2018). Available online at: https://de.statista. com/statistik/daten/studie/161856/umfrage/geburten-nach-dem-alter-dermutter-in-deutschland/ (accessed April 25, 2019).

48. Eurostat. Statistical Office of the European Union Mean Age of Women at Childbirth and at Birth of First Child. (2018). Available online at: https:// ec.europa.eu/eurostat/web/products-datasets/-/tps00017 (accessed April 25, 2019).

49. Eurostat. Statistical Office of the European Union Being Young in Europe Today - Family and Society. (2017). Available online at: https://ec.europa.eu/ eurostat/statistics-explained/index.php/Being_young_in_Europe_today_-family_and_society (accessed April 25, 2019).

50. McDonald FEJ, Patterson P, White KJ, Butow PN, Costa DSJ, Kerridge I. Correlates of unmet needs and psychological distress in adolescent and young adults who have a parent diagnosed with cancer. Psycho-Oncol. (2016) 25:447-54. doi: 10.1002/pon.3942

51. Hauken MA, Senneseth M, Dyregrov A, Dyregrov K. Anxiety and the quality of life of children living with parental cancer. Cancer Nurs. (2018) 41:E1927. doi: $10.1097 /$ NCC. 0000000000000467

52. Krattenmacher T, Kühne F, Führer D, Beierlein V, Brähler E, Resch F, et al. Coping skills and mental health status in adolescents when a parent has cancer: a multicenter and multi-perspective study. J Psychosom Res. (2013) 74:252-9. doi: 10.1016/j.jpsychores.2012.10.003

53. Buchbinder M, Longhofer J, McCue K. Family routines and rituals when a parent has cancer. Fam Sys Health. (2009) 27:213-27. doi: 10.1037/a00 17005

54. Davey MP, Nino A, Kissil K, Ingram M. African American parents' experiences navigating breast cancer while caring for their children. Qual Health Res. (2012) 22:1260-70. doi: 10.1177/1049732312449211

55. Inhestern L, Bergelt C. When a mother has cancer: strains and resources of affected families from the mother's and father's perspective-a qualitative study. BMC Womens Health. (2018) 18:72. doi: 10.1186/s12905-018-0562-8
56. Kissil K, Nino A, Ingram M, Davey M. "I knew from day one that I'm either gonna fight this thing or be defeated": African American parents' experiences of coping with breast cancer. J Fam Nurs. (2014) 20:98119. doi: $10.1177 / 1074840713504035$

57. Patterson P, McDonald FEJ, Butow, P, White KJ, Costa DSJ, et al Psychometric evaluation of the Offspring Cancer Needs Instrument (OCNI): an instrument to assess the psychosocial unmet needs of young people who have a parent with cancer. Support Care Cancer. (2013) 21:192738. doi: $10.1007 / \mathrm{s} 00520-013-1749-\mathrm{z}$

58. Kühne F, Krattenmacher T, Beierlein V, Grimm JC, Bergelt C, Romer G, et al. Minor children of palliative patients: a systematic review of psychosocial family interventions. J Palliat Med. (2012) 15:93145. doi: $10.1089 / \mathrm{jpm} .2011 .0380$

Conflict of Interest: The authors declare that the research was conducted in the absence of any commercial or financial relationships that could be construed as a potential conflict of interest.

Publisher's Note: All claims expressed in this article are solely those of the authors and do not necessarily represent those of their affiliated organizations, or those of the publisher, the editors and the reviewers. Any product that may be evaluated in this article, or claim that may be made by its manufacturer, is not guaranteed or endorsed by the publisher.

Copyright $\odot 2021$ Inhestern, Bultmann, Johannsen, Beierlein, Möller, Romer, Koch and Bergelt. This is an open-access article distributed under the terms of the Creative Commons Attribution License (CC BY). The use, distribution or reproduction in other forums is permitted, provided the original author(s) and the copyright owner(s) are credited and that the original publication in this journal is cited, in accordance with accepted academic practice. No use, distribution or reproduction is permitted which does not comply with these terms. 